\title{
Improving Investor Communications and Analysis via Standardization
}

\author{
Mike Willis. PricewaterhouseCoopers \\ mike.willis@us.pwc.com
}

\begin{abstract}
Over time, a market dialog of taxonomy extension best practices will improve constituent communication and investor analysis and comparability assessments. A stated goal/objective typically associated with the adoption of the Extensible Business Reporting Language $\left(\mathrm{XBRL}^{1}\right)$ is the enhancement of information quality, specifically comparability. Terms often cited include 'Standard', 'Standardization' and 'Comparability'. This paper discusses these three separate but related topics by clarifying common definitions, relationships, uses, and limitations. The paper recognizes various comparability perceptions, and it demonstrates how XBRL enhances comparability and perhaps it takes it to a new level. Finally, the paper suggests continue dialog with market participants on best practices for further development of concepts relevant to enhancing comparability.
\end{abstract}

Key words: XBRL, XBRL taxonomy, XBRL dialog, standards, standardization, comparability, language platform

\footnotetext{
${ }^{1}$ XBRL is the extensible business reporting language, an international information format standard promulgated by an international consortium comprise of over 550 organizations. More information on XBRL can be found http://www.xbrl.org 


\section{INTRODUCTION}

$\mathrm{XBRL}$ is an Internet business standardization language that provides an international reporting platform that enhances communication or dialog among supply chain participants. The dialog among supply chain participants when executed via disciplined taxonomy extension principles will work to enhance the quality, context and comparability of business reported information. Establishing best practices around how and when company-specific extensions to agreed-upon taxonomy concepts are created and used will enhance reported information for comparability purposes.

Allowing companies to extend taxonomies to accommodate company-specific situations is critical to the adoption of XBRL in any business reporting environment. A disciplined extension best practice will maintain the flexibility of the current US accounting structures. The mission of XBRL is NOT to change the accounting standard of any country in which it is used. XBRL must be adapted to the existing accounting structure, enhancing reporting processes and improving information quality.

\section{STANDARD, STANDARDIZATION, AND COMPARABILITY}

Standard - A typical definition for "standard" would be: "An authoritative principle or rule that usually implies a model or pattern for guidance."

In the context of 'business reporting', the phrase 'standard' most often refers to Generally Accepted Accounting Principles (e.g. GAAP ${ }^{2}$ ) or possibly Generally Accepted Auditing Standards (e.g. GAAS). In the context of reporting processes, the phrase 'standard' may also refer to a 'Standard Chart of Accounts' or a 'Standard reporting template' ${ }^{\prime 3}$. Regardless of the type of business reporting standard, its 'general acceptance' is a critical defining criteria. Thus, 'Standard' is a common concept that has many alternative definitions depending upon the context of its use.

In the context of 'business reporting' where 'Standard' most often refers to GAAP, there currently is not a single source of comprehensive definitions for

\footnotetext{
${ }^{2}$ Either US GAAP or IFRS GAAP or other forms of local statutory GAAP

${ }^{3}$ The SEC's 'Financial Data Schedule' was a 'Standard reporting template'. The FDS provided a fixed number of reporting concepts into which registrant information was required to converge or 'fit'. The rule repealing the Financial Data Schedule and related reasons can be found here: http://www.sec.gov/rules/final/33-7855.htm\#P153 44647.
} 
commonly reported concepts. The GAAP 'Standard' is promulgated as reporting 'principles' and does not articulate agreed to definitions for each possible concept that might result from the application of these principles by different companies. For example, , at the present time there are companies that use the exact same phrase/label/term to describe a reported concept and these reported concepts may or may not be comparable.

As an example, reported concepts such as 'Cost of Sales' may be clearly defined by a unique company; however, the reported 'Cost of Sales' of Ford, Intel and General Motors do not conform to any single common definition and/or provide any relevant context for comparability. The 'Cost of Sales' concepts for these three companies certainly do have common components such as salaries, wages, overhead and both fixed and variable costs; however, the label 'Cost of Sales' is properly derived by individual company management based upon their unique business strategy, business model, operations and application of relevant accounting principles. In this instance, is absolute compliance with a single common definition necessary when equivalence with more general categorization may be sufficient?

Even when provided, common phrases, descriptions and even definitions may not always be completely unambiguous and therefore may result in varied perceptions and understandings. Reported concepts can be clear only if the definitions and interrelationships are clear to all parties. While such clarity is useful whether using XBRL or not, later this paper argues that XBRL has a potential to better communicate comparability assessments. XBRL provides a platform for the transparent articulation of definitions and interrelationships critical to an unambiguous understanding.

Standardization - In the business reporting supply chain context, standardization may be defined as: "the process of establishing technical standards for specific business processes that leverage commonly agreed syntaxes."

Standardization does not mean to force all reporting concepts into a standard or fixed template. It means to use agreed syntaxes or a common language to describe reporting concepts; both those common across a range of companies and those unique to a specific company. 
A commonly referred example of a supply chain standardization effort is the Universal Product Code (e.g. 'UPC' or 'bar code') that provides supply chain participants with the ability to describe a product in a standardized manner. The UPC did not require companies to conform to a limited number of product descriptions. The UPC standardized the manner in which products were described so that they could be understood by all supply chain participants. The UPC did not limit the number of products passing through the retail grocery supply chain; rather the standardized barcode dramatically reduced the cost of managing product information and thereby facilitated an exponential increase in the number of supply chain products based upon a wide range of realized process economic efficiencies.

Comparability - Comparability, like other terms, has many alternative definitions including "the assessment of similarities and differences between two concepts". For purposes of this paper, 'Comparability', like art, value and beauty is best determined by the beholder. The intent is not to explicitly define 'comparability' rather to create a supply chain environment in which its assessment is more efficient and effective for both producers and consumers of information.

'Comparability' is often perceived as achieved when two similar concepts are given the 'same' understanding; however, that may not be very useful if it is never the case that concepts are not the same or are different. Knowing that two concepts are really different even when they have similar name/label/term is critical to the comparability value proposition.

'Earning before interest taxes depreciation and amortization' ('EBITDA') is a good example of how comparability can be enhanced via standardization. Many companies report EBITDA, and most of them have their own unique definition. The ability to explicitly describe the company specific definition of EBITDA via the standardized language provides benefits to both preparers and consumers. It also allows the consumer to explicitly understand when reported EBITDA concepts are comparable or when they are similar but not really comparable.

In order to achieve information comparability with any language, there needs to be agreed definitions for common concepts. There are many terms in our English language where there is obvious agreement on definitions; however, the context of the terms used may actually imply a range of agreed definitions. Examples where context is relevant to a full understanding of common concepts include the following phrases: 
- 'Boot' - may mean one thing in a shoe store in North America and something different in a car park in the UK or when calling a computer help desk.

- 'Hood' - may mean one thing in a correctional facility and something completely different in a car dealership.

- 'Gift' - may mean one thing to a child on their birthday and something completely different to parents during an infertility ${ }^{4}$ clinic visit.

We understand the agreed definitions of these terms based upon the context in which they are presented. In the absence of context or sometimes in spite of explicit context, specific definitions may be required to eliminate the possibility of misunderstanding.

Common definitions and context are important to an accurate understanding of common terms in the English language. This is also true within any Internet based semantic 'language' as both providers and consumers need both common definitions and relevant context to ensure that each individual concept is accurately and fully understood.

XBRL is a standardized language that also provides a semantic platform for both relevant context and definitions for business concepts. As a standardized language, it enables specific definitions and contextual concepts but does not inherently provide them. Next section I use common definitions attached to specific semantic labels and explain how standardization enhances comparability; and how the perceived chaos of increasing reporting alternatives through extensions in fact improves information comparability.

\section{BENEFITS OF XBRL AS A STANDARDIZATION LANGUAGE PLATFORM}

While the XBRL technical language can be used to describe a "Standard Chart of Accounts" for a specific organization, it does not require reported concepts to be reported in conformity with any 'Standard' chart of accounts, template or restricted manner of reporting. XBRL Taxonomies are often perceived to be a 'standard' or fixed way of reporting; however, XBRL Taxonomies are 'extensible' and can be

\footnotetext{
${ }^{4}$ GIFT defined via http://www.ivf-infertility.com/gift/gift2.php
} 
expanded by the preparers (and others) to address their individual needs for very specific and unique reporting concepts.

$\mathrm{XBRL}$ is a standardized language platform that enables very clear, concise and accurate articulation of reported concepts; regardless if the concept is part of an agreed public taxonomy or a unique company specific extension thereto. XBRL taxonomies for business reporting concepts are articulated in structured and related hierarchies; thereby enabling taxonomy extensions for unique company concepts to be represented as a 'child' of the public taxonomy 'parent' concept.

Extensibility only makes sense in the context of a recognizable intuitive semantic model. In the case of GAAP reporting concepts, this semantic model is the 'Conceptual Accounting Framework' outlined in FASB Concept $6^{5}$. Broader conceptual models such as Enhanced Business Reporting Framework ${ }^{6}$ or the Global Reporting Initiative G3 Framework7 also provide contextual support for additional non-GAAP concepts. A recognizable conceptual framework provides the context needed for clearer understanding. Clear guidelines for extensibility provide a common understanding of the incremental context required for unique company specific reporting concepts.

The public taxonomy may contain a structure of concepts such as outlined in Table 1:

Table 1.

Taxonomy Hierarchical Structure Example (Conceptual Accounting Framework)

Assets

-----Assets Current

-Cash, Cash Equivalents and Short Term Investments

-Cash and Cash Equivalents

Cash Unrestricted

Company Specific Cash Extension

-Company Specific Cash Extension

Company Specific Cash Extension

-Short Term Investments

\footnotetext{
${ }^{5} \mathrm{http}: / / \mathrm{www}$. fasb.org/pdf/con6.pdf

6 http://www.ebr360.org/ContentPage.aspx?ContentPageId=107

7 http://www.globalreporting.org/ReportingFramework/ReportingFrameworkOverview/
} 
The reporting concept terms in black above are part of the XBRL US GAAP Commercial and Industrial public taxonomy (e.g. part of the commonly recognizable conceptual framework). The reporting concepts in blue represent possible company specific unique extensions. These structures can be used for various semantic relationships within the context of the conceptual framework thereby providing more explicit information. These company specific terms may or may not be 'comparable' with other reporting concepts at this level.

This hierarchical structure provides the context of the public taxonomy concepts as well as extension of these concepts for unique company specific reporting concepts. Companies can extend these taxonomies at any level creating a unique company specific concept. The XBRL Taxonomies provide a standardized manner to describe reported concepts in the context of the hierarchical structure of the conceptual framework.

XBRL does not require companies to conform to the reporting concepts described in the public taxonomy, rather provides a standardized context via the conceptual framework's hierarchical structure that can be used by companies as a low cost starting place that can be extended to meet their unique company specific reporting concept needs. It is expected that companies will use the higher-level reporting concepts and their company-specific concepts will "roll up" to these higher-level concepts. Also, it is expected that companies will likely have taxonomy extensions at the lower levels of the hierarchy more than upper levels; this is consistent with the notion of principles-based reporting standards.

In addition to the standardized taxonomy structure, XBRL provides for the explicit linkage of reporting concepts to other relevant reporting concepts (e.g. accounting principles, policies, statutory regulations, etc.). At the present time, public taxonomies include explicit linkages from individual reporting concepts to relevant and specific GAAP and other relevant regulatory concepts. Currently, there is no explicit linkage from individual reporting concepts to any external form of specific definition for common reporting concepts; however, every US GAAP concept is given a human readable definition to go with the specific label, this adds more explanatory power compared with current paper-based system. Moreover, analyst and investors can link any item in the taxonomy to various reporting models used for comparability assessment. 


\section{ACHIEVING A NEW LEVEL OF COMPARABILITY}

Currently, consumers use the labels/captions applied to reported concepts as a proxy for explicit definitions. Some investors and analysts view company level 'Cost of Sales' concepts as comparable while others seek a more detailed of "Cost of Sales" at business segment level. Others view these common reporting concepts at the company and business segment levels as too high level for comparability and provide their own individual or proprietary adjustments in an effort to achieve comparability. Regardless of the consumers approach, the current assessment of comparability is based primarily on the labels affixed to the reported concepts and the individual consumer's perceptions thereof and any other information provided in the Notes to the financial statements that defines the make-up of the information disclosed.

Difficulties with the current situation is the context of reported concepts provided via a paper centric reporting format limits comparability assessments. The current paper/paper centric reporting platform has resulted in comparability assessments at the highest and most common of reporting concepts (EPS, Gross Margin, EBITDA, etc.) but does not efficiently enable comparability at granular or company specific report concepts. The XBRL format enables an enhanced environment for comparability through the following each of which is outlined below:

1. Improved context

2. Extensibility

3. Explicit definitions

4. Market development of inter-taxonomy mappings

5. Improved modularity and re-use of sets of definitions (dimensions)

6. Continuous dialog

Improved Context - XBRL enables producers and consumers to have very specific context for any reported concepts. Cost of Sales at the company level, business segment, product group, business unit, product type and others are enabled by XBRL for information contained within the company report and/or for the reported concepts that may be individually parsed out of the company reports 
and specifically used by investors/analysts. The complete context of any individual reported concept is retained for immediate use by consumers.

Extensibility - XBRL provides a standardized and extensible platform for producers to provide unique company specific concepts in the context of the overall conceptual accounting framework (and/or other frameworks that may be applicable). The extensible context for unique reporting concepts is useful to both producers and consumers due to the hierarchical taxonomy structure and relationship between common taxonomy "parent" concepts and the unique company specific taxonomy "child" concepts.

The extensibility of XBRL taxonomies allows consumers to make their own individual assessment of reported concepts by assessing comparability at various levels of the hierarchical structure. Consumers can elect to compare an individual unique company specific reported concept at the furthest extended end of the hierarchical structure OR they can elect to aggregate it with other 'child' terms and compare at a 'parent' level further up the hierarchical structure. In this sense 'users' can elect to compare reported concepts at various combined/aggregated and/or individual levels such as those outlined in Table 1.

While 'extensibility' may be good news for preparers interested in telling a unique company specific story, the utility of unrestrained extensibility does tend to negate the consumer's value proposition for XBRL extensions. A key next step is to determine a method/manner to develop constrained, comprehensible, and disciplined extensions that allow software to correctly (even if only partially) process incoming information.

Explicit definitions - XBRL provides a platform for the articulation for unique definitions for reported concepts. Explicit definitions for common reported concepts are currently articulated via the XBRL Label Linkbase for public taxonomy common concepts. Explicit definitions may be provided as incremental content within the Label Linkbase for any incremental unique company specific reported concepts.

As discussed above, explicit definitions are currently not provided as a part of existing reporting principles. Company providers may find it useful to provide explicit definitions for their company specific unique reporting concepts as this is already a common practice with other company specific reported concepts such as 
EBITDA. By providing clear definitions of company-specific extensions through XBRL's linkbase structure, a practice that does not happen today through paperbased system,, company's can better articulate their own unique information and their own unique investment outlook to consumers.

Market development of inter-taxonomy mappings - The relationships between reporting concepts across public taxonomies may also facilitate comparability and highlight areas where convergence should be considered. The alignment of taxonomy framework structures for disparate but related taxonomies should be carefully considered as this may have a significant impact on prospective comparability assessments. Examples of such related taxonomies include the FDIC's bank call report database and International Financial Reporting Standards (IFRS). The ability to relate data between US GAAP reporting and these datasets could prove invaluable to consumers.

Improved modularity and re-use of sets of definitions (dimensions) Establishing reusable information definitions that can be applied across multiple reporting concepts will work to reduce extensions through more explicit context. These 'modular' or 'dimensional' definitions can be applied to one/many reporting concepts to improve their context while also streamlining the need for extensions. Examples of reusable or dimensional definitions could include the following:

- Historical cost, budgeted, projected, restated

- Gross, Net

- $\quad$ Management, Statutory, GAAP

- Before Change in accounting and After Change in accounting

- Segments such as territories, geographies, business units

- Customers such as retail, wholesale, large, small, domestic, and international

- Products such as capital, consumable, financed, non-financed

The value proposition associated with modular or dimensional definitions is found in the reusable nature of the concepts across common and unique company specific taxonomy concepts. 
Continuous dialog - The explicit nature of XBRL taxonomies (both the public taxonomies and company specific unique extensions thereto), transparent and collaborative nature of taxonomy development provides an Internet platform for a continuous dialog among supply chain participants. Tools (e.g. Core Filings' SpiderMonkey ${ }^{8}$ ) enable a public Internet styled (e.g. 'Wikipedia.org') continuous dialog that can rapidly engage and implement definitional concepts for public taxonomy and unique company specific reporting concepts.

These concepts facilitate a more effective supply chain through better information and more accurate context useful to preparers' communications and consumers' assessments.

\section{COMPARABILITY PERCEPTIONS DIALOG}

There are several existing perceptions that this paper attempts to address. Below is a short summary of some of the existing pervasive perceptions that may be false and should be considered in our collaborative planning for the future.

Common vs. Unique - XBRL provides a standardized communication platform in which both common and unique reporting concepts can be accurately expressed and effectively understood and reused. The perception that commonly reported and comparable concepts are critical to effective valuation assessments is false in that unique (rather than common) company attributes are what differentiate valuation assessments.

Comparability delivered - XBRL enables these common definitions and enhanced contextual relationships to occur; however, it is the responsibility of the preparer and consumer communities to engage in a dialog to establish common understandings and meanings. The perception that XBRL delivers 'comparability' through a standard or fixed reporting template is false in the absence of common definitions and enhanced contextual relationships.

Fixed Templates - The perception that XBRL forces a standard or fixed reporting template of common terms is false. Given extensible nature of the web of hierarchically structured taxonomies, as with any freedom in life, there also

\footnotetext{
${ }^{8}$ http://www.corefiling.com/products/spidermonkey.html
} 
comes an inherent responsibility for discipline in reporting as expected by investors in capital markets.

Definitions Provided - Preparers must supplement commonly used labels with explicit definitions and relationships about their unique company specific reporting concepts. The perception that XBRL defines reporting concepts is false in the absence of specific definitions beyond the application of commonly used labels. XBRL provides more explanatory information because it allows commonly used labels in addition to and in connection with any reporting concept.

Preparer Comparability - The perception that comparability is a responsibility borne by preparers in providing reported concepts aligned with a standard or fixed reporting template is false. Because consumers can leverage XBRL taxonomies to conduct their own assessments of comparability based upon the extensible nature of hierarchically structured taxonomies, comparability is a consumer responsibility. Preparers will need to exercise reasonable judgement in the use of company specific extensions, similar to what they do today with paper-based reporting system.

Comparability dictated - The perception that comparability is the responsibility of a single standards board is false in the absence of an ongoing dialog among supply chain participants. Public taxonomies are the foundation for the initiation of the dialog of supply chain participants. A market dialog of taxonomy extension best practices will improve constituent communication and investor analysis and comparability assessments.

\section{RECOMMENDATIONS AND FURTHER RESEARCH}

As XBRL become a world-wide business reporting standard, it is important to engage the public and address various public perceptions. This paper examined public perceptions regarding XBRL standardization and comparability in business reporting. Only through dialog and critical analysis we can address public perceptions of XBRL and its capabilities. In order to drive towards a market environment in which the quality, relevance and comparability of information is enhanced, the following steps are recommended: 
1. Increased education/awareness/understanding as to the nuances associated with 'comparability' and the need for common definitions and enhanced contextual relationships.

2. Increased education/awareness of how the extensible nature of hierarchically structured XBRL taxonomies facilitates communication and therefore comparability of reporting concepts.

3. Apply specific definitions to individual concepts, beyond the application of commonly used labels.

4. Align public taxonomy frameworks so as to enhance prospective comparability and convergence assessments.

5. Increased education/awareness and enhanced tools that enable consumers to more easily conduct their own assessments of comparability based upon the extensible nature of hierarchically structured taxonomies.

6. Create an environment for an enhanced dialog among supply chain participants as to the structure, context and definition of common and/or company specific unique reported concepts.

7. Engage in a dialogue among market participants to work towards agreement on best practices for further development of concepts relevant to enhanced comparability.

There are undoubtedly others that the market dialog will uncover and subsequently address.

Above recommendations include areas for future research and critical analysis of full potentials of XBRL to meet high expectations of users regarding effective communication and comparability assessments. 